\title{
Cephalic access for multi-lead defibrillator therapy is not associated with premature high voltage lead failure
}

\author{
Zaki Akhtar ${ }^{1}$, Idris Harding ${ }^{1}$, Ahmed El Batran ${ }^{1}$, Hanney Gonna ${ }^{2}$, Nilanka Mannakkara ${ }^{1}$, \\ Lisa Leung ${ }^{1}$, Zia Zuberi ${ }^{3}$, Abhay Bajpai ${ }^{3}$, Simon Pearse ${ }^{1}$, Andrew Cox ${ }^{4}$, Anthony Li $^{1}$, Fadi \\ Jourha $^{1}$, Oswaldo Valencia ${ }^{1}$, Zhong $\mathrm{Chen}^{5}$, Manav Sohal ${ }^{6}$, Ian Beeton ${ }^{7}$, and Mark \\ Gallagher ${ }^{2}$ \\ ${ }^{1}$ St George's University Hospitals NHS Foundation Trust \\ ${ }^{2}$ St George's Hospital \\ ${ }^{3}$ University of London St George's Molecular and Clinical Sciences Research Institute \\ ${ }^{4}$ Frimley Park NHS foundation trust, UK \\ ${ }^{5}$ Guys and St Thomas NHS Trust \\ ${ }^{6}$ St. George's University Hospitals NHS Foundation Trust \\ ${ }^{7}$ St Peter's Hospital, Chertsey
}

October 8, 2020

\begin{abstract}
Background: Cardiac resynchronisation therapy-defibrillator (CRT-D) implantation via the cephalic vein is feasible and safe. Recent evidence has suggested a higher implantable cardioverter defibrillator (ICD) lead failure in multi-lead defibrillator therapy via the cephalic route. We evaluated the relationship between CRT-D implantation via the cephalic and ICD lead failure. Methods: Data was collected from three CRT-D implanting centres between October 2008 - September 2017. In total 631 patients were included. Patient and lead characteristics with ICD lead failure were recorded. Comparison of 'cephalic' (ICD lead via cephalic) vs 'non-cephalic' (ICD lead via non-cephalic route) cohorts was performed. Kaplan-Meier survival and a Coxregression analysis were applied to assess variables associated with lead failure. Results: The cephalic and non-cephalic cohorts were equally male $(82.2 \%$ vs $78.3 \%, \mathrm{p}=0.28)$, similar in age $(69.7 \pm 11.5$ vs $68.7 \pm 11.9, \mathrm{p}=0.33)$ and body mass index (BMI) $(27.7 \pm 5.1$ vs $27.1 \pm 5.7, \mathrm{p}=0.33)$. Most ICD leads were implanted via the cephalic vein $(73.7 \%)$ and patients had a median of 2.8 leads implanted via this route. The rate of ICD lead failure was low and similar between both groups $(0.4 \% /$ year vs $0.14 \% /$ year, $\mathrm{p}=0.34)$. Female gender was more common in the lead failure cohort than non-failure ( $50 \%$ vs $18.2 \%$, respectively, $\mathrm{p}=0.01)$ as was hypertension ( $90 \%$ vs $54 \%$, respectively, $\mathrm{p}=0.03$ ). On multivariate Cox regression, female sex ( $\mathrm{p}=0.007)$, hypertension $(\mathrm{p}=0.041)$ and BMI $(\mathrm{p}=0.042)$ were significantly associated with ICD lead failure. Conclusion: CRT-D implantation via the cephalic route is not associated with premature ICD lead failure. Female gender, BMI and hypertension correlate with lead failure.
\end{abstract}

\section{Introduction}

The cardiac resynchronisation therapy-defibrillator (CRT-D) is an established treatment for heart failure which reduces morbidity and mortality (1). The pacing leads are predominantly implanted via the transvenous approach, usually by subclavian or axillary puncture and sometimes by cephalic vein cut-down. There is no standard approach, but cephalic access is feasible, effective and safe (2)(3), irrespective of the number of leads being implanted (4). Traditional lateral subclavian vein puncture has been associated with a higher rate of lead failure than the use of cephalic venous access (5)(6), but a recent report has suggested that 
multi-lead defibrillator therapy utilising the cephalic route is associated with early implantable cardioverter defibrillator (ICD) lead failure (7).

\section{MethodS}

Data was collected retrospectively for patients with a CRT-D implant between October 2008 - September 2017 from three centres. The follow-up duration was determined from implantation to either lead failure, patient death or end of study period. Implantation technique varied amongst the 12 operators. A minority of operators used venogram-guided lateral axillary access as the method of first choice. Cephalic cut down was preferred by most operators and has been reported previously (4). When this vessel was too small to allow access of all the leads, either the axillary or subclavian was used for the remainder. It was at the operator's discretion to decide which lead to place by other routes of access.

Pacing interrogation was performed within 24 hours after implantation, at 6 weeks and subsequently at 6 -month intervals. Patients with defibrillator leads implanted via the cephalic vein were categorised as the 'cephalic' group and patients in whom the defibrillator lead was implanted utilising the subclavian or axillary veins, were categorised as the 'non-cephalic' group.

\section{Lead Failure}

Lead failure was defined as per the Heart Rhythm Society consensus (8). High-voltage leads were considered to have failed if they exhibited: persistent oversensing of non-physiological rapid signals, abnormal impedance in the pace/sense or the shock component, an increase in right-ventricular lead threshold and/or decrease in sensing sufficient to make the lead unreliable (8). All leads that met these criteria were extracted and replaced; all were inspected carefully before and after extraction. Lead extraction for infection and lead revision for displacement were considered separately. Radiological images from the time of implantation were inspected for all leads that subsequently failed.

\section{Statistical analysis}

Continuous variables were conveyed as a mean \pm standard deviation and median with interquartile range (IQR), whilst categorical variables were presented as a number and percentage. Statistical analysis was performed using a Chi-squared test and an independent T-test. A p-value of $<0.05$ was considered significant. Lead longevity was analysed using the Kaplan-Meier model and risk factors were compared using a univariate, multi-variate and Cox regression analysis.

\section{Results}

Over the study period, 631 patients underwent CRT-D therapy and were included in the analysis. In most cases $(73.7 \%)$, the high-voltage lead was implanted via the cephalic vein (cephalic group). Both groups were pre-dominantly male $(82.1 \%$ vs $78.3 \%$, respectively, $\mathrm{p}=0.28)$ of a similar age $(69.7 \pm 11.5$ years vs $68.7 \pm 11.9$, respectively, $\mathrm{p}=0.33)$ and had a left sided implant $(96.3 \%$ vs $97 \%, \mathrm{p}=0.7)$ for primary prevention $(91.2 \%$ vs $94 \%, \mathrm{p}=0.26$ ). Co-morbidities in both groups were fairly similar (table 1 ) although chronic kidney disease was more prevalent in the cephalic group $(16.6 \%$ vs $9.6 \%$, respectively, $\mathrm{p}=0.03)$. The mean follow-up period was $4.75 \pm 2.4$ years. The overall lead failure rate in this study was $0.33 \% /$ year.

During the study period, 20 patients required revision or replacement of the RV lead. Of these, $6(30 \%)$ patients had an infection indication, including erosion, local infection, and systemic sepsis. Early lead replacement for displacement or cardiac perforation accounted for $4(20 \%)$ cases and the remaining $10(50 \%)$ were premature lead failures.

High-voltage lead failure was rare; failure occurred at a rate of $0.4 \%$ per year in the cephalic group and $0.14 \%$ per year in the non-cephalic group ( $\mathrm{p}=0.34$; figure 1$)$. The number of shock coils, the number of concomitant leads implanted with the defibrillator lead and the ICD lead tip position within the right ventricle did not affect lead longevity (figure $\mathbf{1}$ ). However, ICD leads implanted in female patients for CRT-D, were more likely to experience premature failure $(\mathrm{p}=0.01)$ (figure $\mathbf{2}$ ). 


\section{Comparison of lead failure and non-failure cohorts}

Comparison of the ICD lead failure and non-failure cohorts was performed for baseline patient characteristics (table 2) . There was a significantly higher proportion of female ( $50 \%$ vs $18.8 \%, \mathrm{p}=0.01)$ and hypertensive patients $(90 \%$ vs $54 \%, \mathrm{p}=0.03)$ in the lead failure group. There was a trend toward patients of higher body mass index (BMI) (31.7 vs 27.4, $\mathrm{p}=0.17$ ) and toward a longer implantation procedure duration (139.1 vs 126.5 minutes, $\mathrm{p}=0.62)$ in the lead failure group. A similar proportion of ICD leads were implanted via the cephalic in lead failure and non-failed groups ( $90 \%$ vs $72 \%, \mathrm{p}=0.24)$. When the ICD lead was implanted via the cephalic vein, a statistically similar number of leads were implanted via this route concomitantly, in both cohorts (2.89 vs 2.83 , lead failure vs non-failure, respectively, $\mathrm{p}=0.59$ ).

\section{Predictors of Lead failure}

A univariate logistic regression analysis of the whole study population was performed for predictors of lead failure (table3 ). Female gender ( $\mathrm{p}=0.02$, OR 4.42, [1.258-15.51]) and BMI ( $\mathrm{p}=0.03$, OR 1.12 [1.01-1.24]) were significant factors whilst hypertension was strongly correlated although it did not reach statistical significance $(\mathrm{p}=0.055$, OR $7.62[0.96-60.5])$. When entered in to a Cox regression analysis, female gender $(\mathrm{p}=0.007 \mathrm{OR}$ 7.32 [1.7-31.1]), hypertension ( $\mathrm{p}=0.041$, OR 9.96 [1.1-90.5]) and BMI ( $\mathrm{p}=0.042$, OR 1.12 [1.004-1.24]) were significant predictors of lead failure.

\section{Discussion}

The current series is the largest to date evaluating the relationship between multi-lead defibrillator therapy delivered via the cephalic vein, and ICD lead failure. We found a very low incidence of lead failure in this solely CRT-D based study (0.33\%/year). The findings have important practical applications as CRT-D system implantations via the cephalic are efficient and safe (4), whilst ICD lead failures maintain a degree of concern (7).

This multi-centre study reported a very low overall lead failure rate which is at odds with some previous series (7) but is validated by at least one previous large series $(0.45 \% /$ year $)(9)$. This low failure rate may reflect our conservative practice: A policy of concentrating on products with a track record of long-term safety and late adoption of less tested technology. The higher incidence of failure in the prior literature may represent a publication bias; it is reasonable to suppose that colleagues are more likely to report an unsatisfactory experience than to describe lead performance that is in line with expectation.

This series demonstrates that cephalic vein access for multi-lead defibrillator therapy does not affect lead longevity: The rate of lead failure was similarly low for cephalic and non-cephalic routes $(0.4 \% /$ year vs $0.14 \% /$ year, $\mathrm{p}=0.34$ ). This is in stark contrast to a recent report by Barbhaiya et al which found that cephalic access was associated with a high rate of lead failure in multi-lead ICD therapy (11\% per year for non-Linox and $19 \%$ per year for Linox leads) (7). There are significant differences between the two reports. Their method was to implant a maximum of two leads via the cephalic vein, whereas most of our patients received three leads by this route. Barbhaiya et al described only $46 \%$ of their cohort as having multi-lead ICD systems, our study population consists entirely of CRT-D devices ([?] 2 leads) and they implanted only $18 \%$ of ICD leads via the cephalic vein, while we used it in $74 \%$. This implies that their series included only around 55 ICD leads implanted via the cephalic as part of a multi-lead system compared to 465 in our series.

Sample size alone cannot account for the contrast between our results and those of Barbhaiya et al. As their series included just 6 instances of lead failure including $4(67 \%)$ implanted via the cephalic route, the association may have been a chance event detected on post-hoc analysis. Inter-institution differences in implantation technique could also have played a role: Barbhaiya et al demonstrated that the phenomenon they described was not attributable to a single operator (10), but institutional culture determines the idiosyncrasies of operative technique as much as inter-individual variation. All of the predominantly cephalic operators in our series derived at least part of their methodology from one mentor.

We believe that many small technical (11) and methodological differences could play a role in lead durability: for example, our policy is to place all leads via peel-away sheaths to protect the tip from stress produced 
by passage through a tortuous cephalic vein. In our series, $75 \%$ of the operators would be considered as 'cephalic-operators' with a similar well-honed technique and experience in accessing this vein, maintaining consistency and minimising error. Our population also had a low proportion of leads that have exhibited a high rate of failure such as the Linox (Biotronik, Berlin, Germany).

The Cox regression analysis found that venous access route does not predict lead failure, in keeping with prior reports (12). Consistent with previous findings, we found leads implanted in women were much more likely to fail (13) (figure $\mathbf{2}$ ). The naturally smaller female frame may enforce tighter angulation within the thoracic vasculature, applying stress on the implanted leads. Due to their smaller size, women are also more likely to have excess redundant lead folded within the pocket, increasing lead tension at this site.

Hypertension emerged as an independent risk factor for lead failure in our series, but has previously not been identified as a predictor. It stands to reason as a hazard to lead durability: Hypertension results in shear stress on the vascular system leading to remodelling with increased tortuosity and angulations in the arterial system. The venous system is not directly altered by arterial hypertension, but the close anatomic relationship could expose venous leads indirectly to the angulations of the associated arteries.

\section{Limitations}

This study was a retrospective analysis and therefore open to bias from confounding variables. Due to a relatively smaller sample size for non-cephalic access, propensity matching could not be performed.

\section{Conclusion}

Multi-lead ICD therapy delivered via the cephalic vein is associated with a low risk of lead failure in the long-term. Our data confirm that female gender is a predictor of lead failure and suggest that hypertension may be a previously unidentified risk factor.

\section{REFERENCES}

1. Moss AJ, Hall WJ, Cannom DS, Klein H, Brown MW, Daubert JP, et al. Cardiac-Resynchronization Therapy for the Prevention of Heart-Failure Events. N Engl J Med. 2009 Oct;361(14):1329-38.

2. Ussen B, Dhillon PS, Anderson L, Beeton I, Hickman M, Gallagher MM. Safety and Feasibility of Cephalic Venous Access for Cardiac Resynchronization Device Implantation: CEPHALIC ACCESS FOR CRT IMPLANTATION. Pacing Clin Electrophysiol. 2011 Mar;34(3):365-9.

3. Hadjis A, Proietti R, Essebag V. Implantation of cardiac resynchronization therapy devices using three leads by cephalic vein dissection approach. EP Eur. 2017 Sep 1;19(9):1514-20.

4. Harding I, Mannakkar N, Gonna H, Domenichini G, Leung L, Zuberi Z, et al. Exclusively cephalic venous access for cardiac resynchronisation: A prospective multi-centre evaluation. Pacing Clin Electrophysiol. 2020 Aug 28;pace.14046.

5. Roelke M, O'Nunain SS, Osswald S, Garan H, Harthorne JW, Ruskin JN. Subclavian Crush Syndrome Complicating Transvenous Cardioverter Defibrillator Systems. Pacing Clin Electrophysiol. 1995 May;18(5):973-80.

6. Chan N-Y, Kwong N-P, Cheong A-P. Venous access and long-term pacemaker lead failure: comparing contrast-guided axillary vein puncture with subclavian puncture and cephalic cutdown. Europace. 2016 Oct 12;euw147.

7. Barbhaiya CR, Niazi O, Bostrom J, Patil S, Jankelson L, Bernstein S, et al. Early ICD lead failure in defibrillator systems with multiple leads via cephalic access. J Cardiovasc Electrophysiol. 2020 Jun;31(6):1462-9.

8. Kusumoto FM, Schoenfeld MH, Wilkoff BL, Berul CI, Birgersdotter-Green UM, Carrillo R, et al. 2017 HRS expert consensus statement on cardiovascular implantable electronic device lead management and extraction. Heart Rhythm. 2017 Dec;14(12):e503-51. 
9. Eckstein J, Koller MT, Zabel M, Kalusche D, Schaer BA, Osswald S, et al. Necessity for Surgical Revision of Defibrillator Leads Implanted Long-Term: Causes and Management. Circulation. 2008 May $27 ; 117(21): 2727-33$.

10. Barbhaiya CR, Niazi O, Jankelson L, Bernstein S, Park D, Holmes D, et al. Response to: Do not yet abandon cephalic vein access for multiple leads in ICD implantation. J Cardiovasc Electrophysiol. 2020 Oct;31(10):2789-90.

11. Maass AH, Groenveld HF, Rienstra M. Do not yet abandon cephalic vein access for multiple leads in ICD implantation. J Cardiovasc Electrophysiol. 2020 Oct;31(10):2788-2788.

12. Birnie DH, Parkash R, Exner DV, Essebag V, Healey JS, Verma A, et al. Clinical Predictors of Fidelis Lead Failure: Report From the Canadian Heart Rhythm Society Device Committee. Circulation. 2012 Mar 13;125(10):1217-25.

13. Padfield GJ, Steinberg C, Karim SS, Tung S, Bennett MT, Le Maitre JP, et al. Early Failure of the Biotronik Linox Implantable Cardioverter Defibrillator Lead: Early Failure of the Biotronik Linox. J Cardiovasc Electrophysiol. 2015 Mar;26(3):274-81.

\section{FIGURE LEGENDS}

Figure 1: Kaplan-Meier curves describing lead survival.A) No significant difference in survival of ICD leads was detected between those implanted via the cephalic and non-cephalic routes. B) ICD lead implanted in septal and apical locations lasted equally well. C) Durability of the ICD lead was not influenced by the number of leads implanted via the cephalic vein.D) The number of coils of the implanted ICD lead does not affect lead longevity.

Figure 2 : Kaplan-Meier curve of ICD lead survival by gender. The rate of defibrillator lead survival was significantly poorer in female patients, compared to their male counterparts $(\mathrm{p}=0.01)$.

Figure 3: Examples of radiographic images from fluoroscopy stored at the time of implantation for patients who subsequently experienced lead failure. The numbering of patients corresponds to the order of implantation and the numbering in table 4. A)Corresponding to patient 1. B) Corresponding to patient 3.C) and D) Corresponding to patient number 4.E) Corresponding to patient 5. F) Patient number 6.G) Patient 7. H) Patient number 8 with tortuosity of the innominate vein I) Venogram of Patient 9 demonstrating a large cephalic vein (dotted arrow) which was not used; instead the system was implanted by lateral axillary puncture (solid arrow).J) Corresponding to patient 10.

\begin{tabular}{llll}
\hline & ICD lead 'cephalic' & ICD lead 'non-cephalic' & p-value \\
\hline $\mathrm{N}=$ & $465(73.7 \%)$ & $166(26.3 \%)$ & $<\mathbf{0 . 0 0 1}$ \\
Male & $382(82.2 \%)$ & $130(78.3 \%)$ & 0.28 \\
Age & $69.7 \pm 11.5$ & $68.7 \pm 11.9$ & 0.33 \\
Number of leads & $2.8 \pm 0.32$ & $2.6 \pm 0.64$ & $<\mathbf{0 . 0 0 1}$ \\
BMI & $27.7 \pm 5.1$ & $27.1 \pm 5.7$ & 0.33 \\
Ischaemic cardiomyopathy & $347(74.6 \%)$ & $123(74.1 \%)$ & 0.89 \\
IHD & $338(72.7 \%)$ & $118(71.1 \%)$ & 0.69 \\
Diabetes & $117(25.2 \%)$ & $49(29.5 \%)$ & 0.27 \\
CKD & $77(16.6 \%)$ & $16(9.6 \%)$ & $\mathbf{0 . 0 3 1}$ \\
Hypertension & $255(54.8 \%)$ & $91(54.8 \%)$ & 0.997 \\
Atrial fibrillation & $170(36.6 \%)$ & $52(31.3 \%)$ & 0.23 \\
LVEF (\%) & $29.1 \pm 13.2$ & $28.3 \pm 8.6$ & 0.42 \\
Procedure (minutes) & $120.6 \pm 46.6$ & $143.9 \pm 44.8$ & $<0.0001$ \\
RV lead failure & $\mathbf{9}(1.94 \%)$ & $\mathbf{1}(0.6 \%)$ & 0.24 \\
Lead follow-up (days) to failure, death or study end & $1788 \pm 914.4$ & $1574 \pm 744.3$ & $\mathbf{0 . 0 0 3 1}$ \\
Left sided implant & $448(96.3 \%)$ & $161(97 \%)$ & 0.7
\end{tabular}




\begin{tabular}{llll}
\hline & ICD lead 'cephalic' & ICD lead 'non-cephalic' & p-value \\
\hline Primary prevention & $424(91.2 \%)$ & $156(94 \%)$ & 0.26 \\
\hline
\end{tabular}

Table 1: A comparison of the cephalic and non-cephalic groups. On average a statistically significant higher number of leads were implanted via the cephalic, than non-cephalic routes. The procedure was also noted to be longer in the non-cephalic procedures. However, there was no significant difference in the number of ICD lead failures between the two groups $(\mathrm{p}=0.24)$.

\begin{tabular}{llll}
\hline & Lead failure & Lead non-failure & p-value \\
\hline $\mathrm{N}=$ & 10 & 621 & \\
Age & $65.5 \pm 10.3$ & $69.5 \pm 11.6$ & 0.26 \\
Female & $50 \%$ & $18.2 \%$ & $\mathbf{0 . 0 1}$ \\
Implantation procedure duration (mins) & $139.1 \pm 77.6$ & $126.5 \pm 46.6$ & 0.62 \\
Ischaemic Heart Disease & $60 \%$ & $72.9 \%$ & 0.36 \\
Diabetes & $20 \%$ & $26.6 \%$ & 0.64 \\
Chronic Kidney Disease & 0 & $15.0 \%$ & 0.19 \\
Hypertension & $90 \%$ & $54 \%$ & $\mathbf{0 . 0 3}$ \\
Atrial fibrillation & $30 \%$ & $35.4 \%$ & 0.72 \\
Body Mass Index & $31.7 \pm 8$ & $27.4 \pm 5.2$ & 0.17 \\
Left Ventricular Ejection Fraction (\%) & $26.1 \pm 5.7$ & $28.4 \pm 28.4$ & 0.33 \\
Lead age to failure or end of follow-up (days) & $1433.9 \pm 922.4$ & $1738.2 \pm 878.1$ & 0.33 \\
Cephalic access (\%) & $90 \%$ & $73.4 \%$ & 0.24 \\
Number of cephalic leads when ICD lead cephalic & $2.89 \pm 0.3$ & $2.83 \pm 0.4$ & 0.59 \\
\hline
\end{tabular}

Table 2: A comparison of the defibrillator lead failure and non-failure patients.

Abbreviation: ICD, implantable cardioverter defibrillator

Table 3: Regression analyses for variates of lead failure

\begin{tabular}{lll}
\hline Variable & Odds ratio & $\mathbf{9 5 \%}$ CI \\
\hline Univariate binary logistic regression analysis & Univariate binary logistic regression analysis & Univariate bi \\
Female gender & 4.417 & $1.258-15.511$ \\
Body mass index & 1.123 & $1.011-1.246$ \\
Hypertension & 7.615 & $0.959-60.47$ \\
Diabetes mellitus & 1.438 & $0.302-6.84$ \\
Cephalic ICD lead access & 3.37 & $0.424-26.8$ \\
Total number of leads & 0.87 & $0.108-6.994$ \\
Multivariate regression analysis & Multivariate regression analysis & Multivariate \\
Female gender & 6.769 & $1.522-30.101$ \\
Hypertension & 8.092 & $0.936-69.983$ \\
Body mass index & 1.106 & $0.992-1.233$ \\
Cox regression analysis & Cox regression analysis & Cox regressio \\
Female gender & 7.316 & $1.724-31.049$ \\
Hypertension & 9.963 & $1.096-90.54$ \\
Body mass index & 1.117 & $1.004-1.243$ \\
\hline
\end{tabular}

Abbreviation: ICD, implantable cardioverter defibrillator 


\begin{tabular}{lllllll}
\hline Patient & Prevention & Gender & Age & Co-morbidities & BMI & Number of leads via the cephalic \\
\hline 1 & Secondary & M & 79 & AF, HTN & 30 & 3 \\
2 & Secondary & M & 70 & IHD, HTN, IBD & 22 & 2 \\
3 & Secondary & F & 52 & IHD, DM, RA, Asthma, HTN & 35 & 3 \\
4 & Secondary & F & 63 & DCM, HTN & 36 & 3 \\
5 & Primary & F & 71 & IHD, HTN, COPD & 29 & 3 \\
6 & Primary & F & 46 & IHD, IBD & 48 & 3 \\
7 & Primary & M & 75 & DCM, HTN, AF & 30 & 3 \\
8 & Primary & M & 73 & DCM, HTN & 27 & 3 \\
9 & Primary & F & 64 & IHD, HTN, , HT, DM & 26 & 0 \\
10 & Primary & M & 62 & IHD, HTN, AF, DM & 33 \\
\hline
\end{tabular}

Table 4: Summary of the premature ICD lead failure cohort. Patients are numbered chronologically according to the time of initial implant.

Abbreviations: ICD, implantable cardioverter defibrillator

F: Female

M: Male

AF: Atrial Fibrillation

HTN: Hypertension

IHD: Ischaemic Heart DIsease

IBD: Inflammatory Bowel Disease

DM: Diabetes Mellitus

DCM: Dilated Cardiomyopathy

COPD: Chronic Obstructive Pulmonary Disease
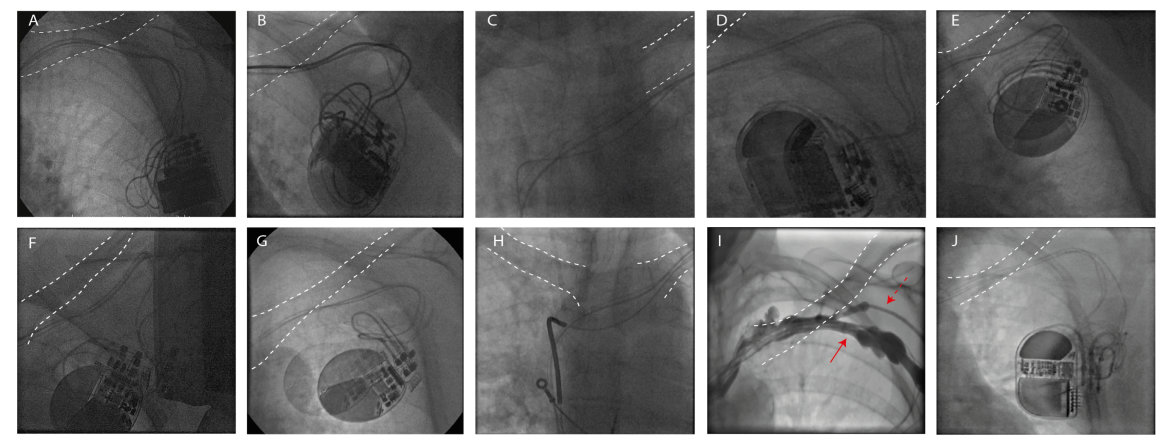


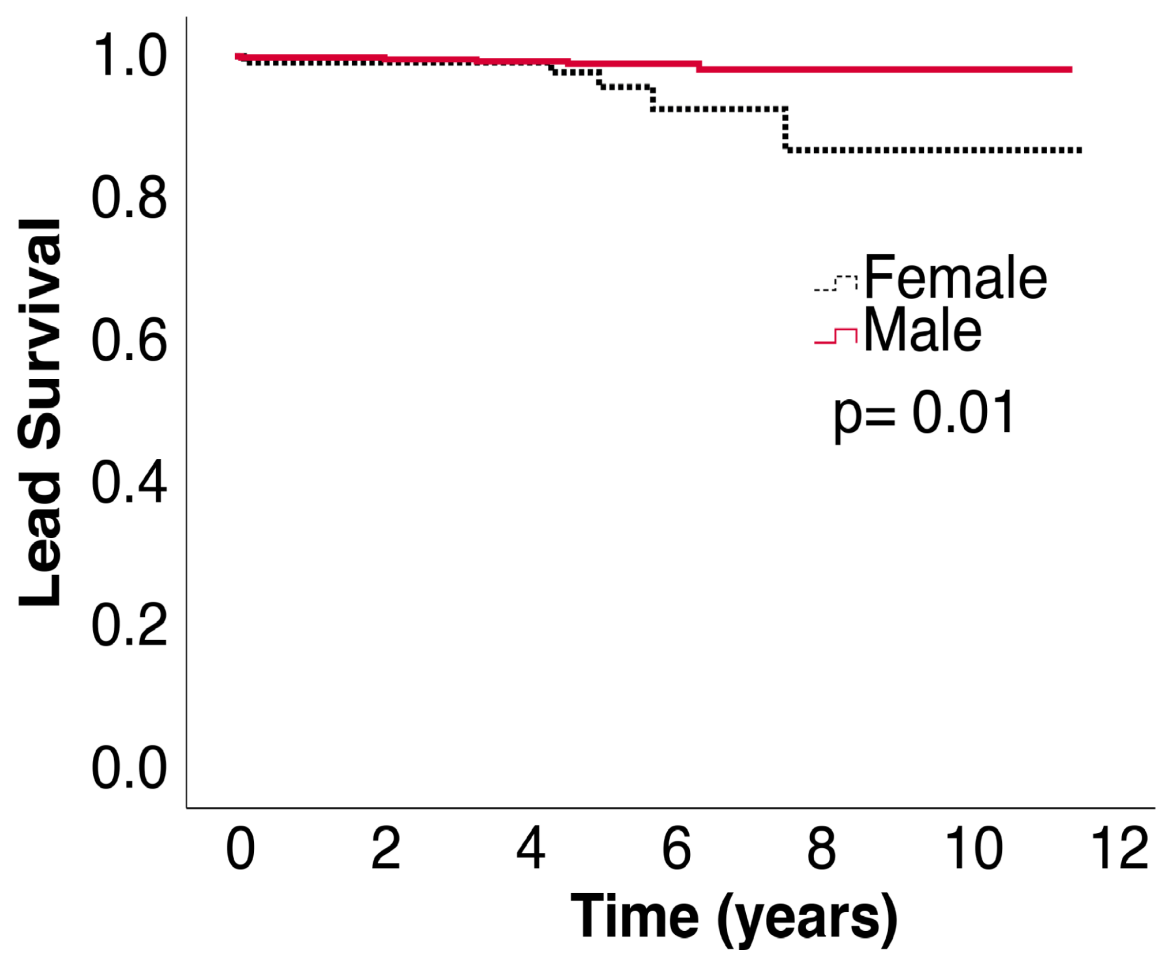

A

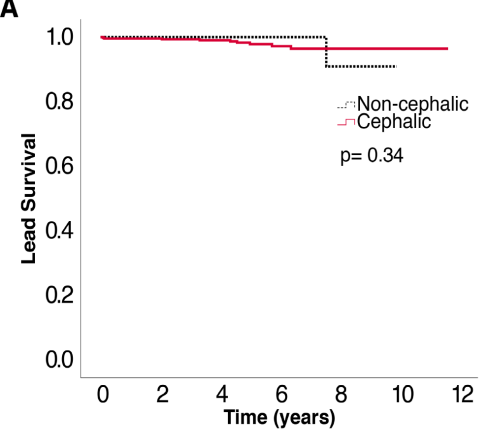

C

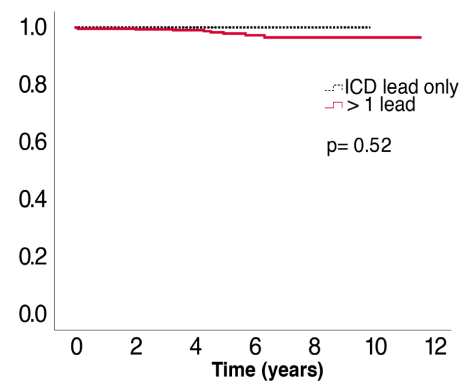

B

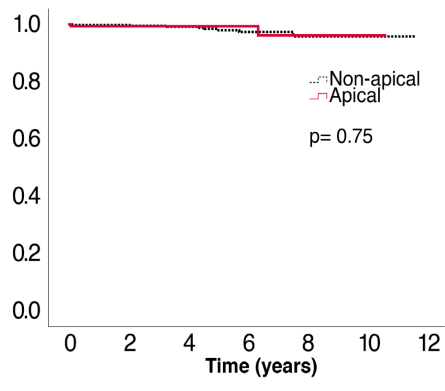

D

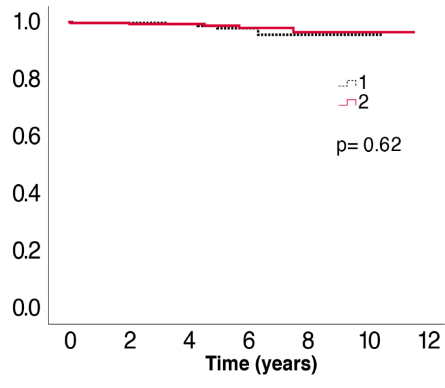

